\title{
Fille en rouge hurlant
}

\section{Michel Sandras}

\section{(2) OpenEdition \\ Journals}

Édition électronique

URL : http://journals.openedition.org/ccs/1038

DOI : $10.4000 /$ ccs. 1038

ISSN : 2558-782X

\section{Éditeur :}

Presses universitaires de Rennes, Association des lecteurs de Claude Simon

\section{Édition imprimée}

Date de publication : 1 décembre 2017

Pagination : 113-127

ISBN : $978-2-7535-5482-5$

ISSN : $1774-9425$

Référence électronique

Michel Sandras, «Fille en rouge hurlant », Cahiers Claude Simon [En ligne], 12 | 2017, mis en ligne le 07 décembre 2018, consulté le 13 février 2020. URL : http://journals.openedition.org/ccs/1038 ; DOI 10.4000/ccs. 1038 


\title{
FILLE EN ROUGE HURLANT
}

\author{
Michel SANDRAS \\ Université Denis Diderot-Paris 7
}

"Écrire ce qu'on a vu est en réalité le façonner " (M. Merleau-Ponty à propos de Claude Simon, dans Notes de cours, Gallimard, 1996, p. 218)

La scène du "coït aérien ", située dans l'épisode qui ouvre le chapitre vi de L'Acacia ${ }^{1}$ intitulé " 27 août 1939 », a pour motif la séparation des amants quand l'homme part pour la guerre: le roman, la poésie, la chanson, la photographie, le cinéma l'ont largement illustré. Je voudrais montrer la place singulière qu'elle tient dans le livre. Fille en rouge hurlant: ce titre, moins pour en évoquer un autre, cher aux peintres et aux sculpteurs - comme par exemple Femme piquée par un serpent, bien que le marbre d'Auguste Clésinger, à l'époque (1847) jugé scandaleux à cause de la posture paroxystique de la femme, ne soit pas totalement hors-sujet - que pour signaler l'absence de l'article, qui ferait de la chose vue une allégorie. Ce que j'essaierai d'argumenter après avoir examiné comment les modes de figuration des corps permettent de distinguer ou de rapprocher certaines scènes (dans L'Acacia et dans d'autres romans de Claude Simon).

\section{LA GRAPPE, LE GRAPHE}

Les pages qui ouvrent le chapitre montrent la foule rassemblée dans la gare: la foule, ou plutôt le conglomérat humain d'où s'extrayaient l'une après l'autre de nouvelles silhouettes se hissant sur les marchepieds, semblait se resserrer plus étroitement, comme si

1. Les réservistes sont rappelés par tranches dès le lendemain de l'annonce du pacte germano-soviétique, soit à partir du 23 août. Rappelons que la date du 27 août est celle de la mort du père dans la guerre précédente et que le train va prendre la même direction, celle de l'est de la France. 
non seulement il refusait de se désagréger, de se laisser entamer, mais encore se rétractait pour ainsi dire sur lui-même, les particules qui le composaient comme soudées les unes aux autres dans une sorte de cristallisation, une inextricable multitude d'étreintes non pas, pour la plupart, de deux corps mais de plusieurs, assemblés en couronnes autour de centres où parvenaient des voix étranglées de femmes et des pleurs d'enfants, de sorte que ceux qui s'en arrachaient [...] étaient encore retenus, empêchés de progresser ou parfois même repoussés, éloignés des marchepieds par quelque poussée, quelque convulsion ou dilatation du grouillement bigarré, incohérent, parcouru d'imprévisibles courants, d'imprévisibles reflux. [...] [L]a marée humaine [...] était venue [...] se coller ou plutôt adhérer avec quelque chose de furieux, d'obstiné et de gluant au flanc des wagons ( $A$, p. 1103)

Cette représentation à la fois topologique et moléculaire est présente dans toute l'œuvre de Claude Simon ${ }^{2}$. Représentation topologique, qui met l'accent sur la déformation: une forme multiple subit des phénomènes successifs d'agrégation et de désagrégation, de rétraction et de dilatation. Moléculaire parce qu'elle souligne la composition en particules. La métaphore des particules ou celle de l'agglutination ou de la concrétion rendent sensible un mode d'agglomération suscitant un léger effet de dégoût, comme on le trouvait déjà à la fin du chapitre $\mathrm{V}$ (l'arrivée du bateau ${ }^{3}$ ). Le lexique des lignes précédant juste notre passage confirme cette impression: l'espèce d'hétéroclite « conglomérat " d'hommes et de femmes, les détritus à la surface d'une eau trouble, les "grappes humaines ", représentations qui seront aussi celles de l'exode, de la fuite des civils:

le double chapelet d'épaves que semblait avoir laissé sur ses bords après s'être retirée quelque rivière en crue charriant pêle-mêle véhicules, bêtes et gens repoussés sur les côtés, parfois isolés, d'autres fois agglutinés, comme arrêtés par quelque obstacle, enchevêtrés au milieu du contenu éparpillé et blafard (linges, courtepointes, journaux froissés), de ballots ou de valises crevées (p. 1193)

L'association des bruits ("fracas de tampons et d'attelages entrechoqués, comme hoquetant ») et de la perception d'une multiplicité grouillante ("à la façon d'un animal couvert de parasites ") réapparaitra plus loin pour comparer le piétinement des chevaux aux bruits que font les insectes:

2. Voir dans Les Corps conducteurs: "les mêmes particules [...] recomposent inlassablement un autre ensemble à la fois différent et en tous points pareil au précédent ", contrairement aux " mouvements de foule concertés (processions, cortèges, manifestations politiques)» (CC, p. 437).

3. «Elle ne voit pas la foule. Elle voit un confus amas de particules multicolores, claires ou foncées, agglutinées et mouvantes » (p. 1100). Il est juste de signaler que la représentation moléculaire peut parfois être d'ordre poétique: «les vols d'étourneaux étiraient leurs écharpes, tournoyaient, se rassemblaient, se condensaient en soleils charbonneux, puis explosaient aurait-on dit, se déployaient de nouveau en myriades d'infimes et palpitantes particules» (p. 1136). Voir aussi p. 1062: "Il ne voit pas les infimes particules de diamant laissées par la rosée sur la partie du pré encore à l’ombre de la haie. » 
grésillement semblable (avec parfois quelques cliquetis, quelques tintements, de légers froissements de métal entrechoqué, comme des froissements d'élytres, les claquements de corselets ou de mandibules) à la confuse rumeur de myriades d'insectes s'abattant en d'obscures nuées [...] se montant les uns sur les autres (p. 1159)

Dans tous nos exemples domine la série énumérative ${ }^{4}$, renforcée souvent de répétitions lexicales, parfois structurée par des constructions anaphoriques. Dans L'Acacia, elle est présente à de nombreuses reprises et sous de multiples aspects, par exemple dans l'évocation de la chair morcelée sous l'effet du plaisir, comme dans l'épisode du bordel:

quelque chose de vivant, mobile: crins, muqueuses, lèvres, salive, langues, yeux, voix, souffles: la chair sans mensonge, crédible, docile dans ses mains, se mouvant, s'écartant, s'ouvrant: la solitude, la mort, le doute conjurés, vaincus (p. 1241)

Si la scène du " coït aérien " s'élève sur fond de "conglomérat humain ", c'est pour s'opposer à cette représentation topologique et moléculaire de la grappe. Le premier indice est la reformulation " non pas cramponnée mais tenue à bras-le-corps " : à la sensation de la colle, du " gluant " caractérisant la foule est substituée la vue d'un couple qui tient par la force physique de l'un et l'audace ardente de l'autre. L'agglomération molle qui suscite la répugnance fait place à la figure érotique et acrobatique de l'arc, la grappe à un hiéroglyphe.

À partir de la locution " De sorte que » jusque la fin de l'alinéa (« de plus en plus vite»), se déploie une seule phrase dont la construction syncopée rend compte d'un affolement: celui du témoin penché à la fenêtre qui mesure le danger couru par la jeune femme au moment où le train prend de la vitesse, celui de l'écrivain soucieux de donner au lecteur les étapes d'une séparation jusqu'au climax, au court moment qui totalise une situation, des gestes et des cris, y compris la façon équivoque dont on le sent affecté par eux: le pathétique est altéré par la présence de comparaisons crues ("ventouse ", " coït ", " copuler »). La structure syntaxique est remarquable par le concours de deux modèles presque opposés. Le premier, canonique selon la norme narrative, est articulé par des propositions temporelles qui assurent au texte enchaînement et profondeur. C'était le modèle dominant dans le début de l'épisode qui montrait l'arrivée lente du train: exemple de phrase "profonde ", assez classique, par le développement d'une protase consistante antéposant les compléments et d'une apodose cimentée par des

4. Nous renvoyons à notre étude "Multiplicités: la série énumérative dans L'Acacia", Cahiers Claude Simon, $\mathrm{n}^{\circ} 11$, PUR, 2016, p. 147-159. 
relatives et des conjonctives, fermée par la série de trois adjectifs "quelque chose d'irrémédiable, définitif et terrible»(p. 1101). Alors qu'ailleurs dans L'Acacia la construction des suites énumératives est le plus souvent asyndétique (les unités qui les composent sont séparées seulement par des virgules), ici apparaissent des adverbes (puis, toujours, enfin) qui indiquent un ordre temporel de gestes qui épouse le départ du train et la vitesse qu’il acquiert, la course de la jeune femme, son arrêt forcé. Le second modèle, celui-là propre à Claude Simon, est remarquable par la déliaison et la pure succession " horizontale " de séries ayant pour pivots des participes passés et surtout présents: c'est celui qui souligne le motif du chaos. Alors que souvent les séries sont continues, ne s'y intercalant que des indices de reformulation (" ou plutôt ", " non pas... mais ») ou de comparaison ("comme si »), ici existe une rupture de construction très visible:

[sa légère robe] soulevée par l'air, puis claquant sur les cuisses, des cris d'effroi s'élevant, et un moment elle parut pour ainsi dire suspendue dans le vide $[\ldots]$ une gerbe de mains se tendant vers elle, la tirant (ou la recevant), le train roulant maintenant (p. 1104)

Déséquilibre de la phrase, assez rare dans ce roman, qui mime la position asymétrique du couple. L'accumulation des participes passés et présents attire aussi l'attention: bien que ponctuant une chronologie de gestes et s'inscrivant dans un enchainement, elle efface en fait les repères qui pourraient orienter l'action en ne retenant que la scansion, les effets de battement, ce qui à la fois sépare et unit.

\section{MOUVEMENTS, POSTURES}

La figuration des corps des amants prend en compte ou leur déplacement dans le champ de vision d'un observateur (le modèle est cinématographique, mais les romanciers du $\mathrm{XIX}^{\mathrm{e}}$ siècle l'avaient déjà illustré) ou la saisie d'une posture (le modèle est pictural, sculptural, photographique). La première figure est celle de l'apparition (ou de la disparition) progressive d'un personnage ou d'un objet à partir de la perception de celui qui le voit: on peut dire que le passage du proche au lointain (ou l'inverse) donne une vision du temps ${ }^{5}$. Pour la préciser s'impose le vocabulaire du cinéma qui l'a

5. L'apparition (ou la disparition progressive) est un motif récurrent chez Claude Simon. Elle a son équivalent sonore, dans le phénomène d'augmentation ou de diminution d'un bruit - le crescendo ou le diminuendo des partitions musicales: par exemple dans La Route des Flandres et L'Acacia le bruit de la pluie et des sabots des chevaux, qui est en quelque sorte le bruit du temps. 
largement utilisée. On peut l'obtenir soit par un travelling (arrière ou avant) lent qui correspond au déplacement de celui qui voit, l'objet restant immobile, soit par un plan fixe qui fait apparaître ou disparaître progressivement le personnage dans la profondeur du champ. Cette figure est lyrique par la place qu'elle donne à l'attente, à l'horizon, à la rêverie ${ }^{6}$. Ici la clausule de l'épisode souligne la disparition progressive de la « silhouette perdue dans le soleil d'août au milieu des aiguillages, des rails étincelants » en l'inscrivant dans un espace cosmique. Description reprise quelques pages plus loin, qui exacerbe l'amenuisement:

des taches - puis même plus des taches: des points - puis même plus des points: rien qu'un indistinct et sombre agrégat sur le quai de la gare miniature perdu au milieu de l'océan des vignes dans l'éblouissante lumière d'août, aspiré en arrière, s'éloignant, rapetissant encore, puis, à son tour, comme un peu plus tôt la verrière béante, la fille en rouge hurlant, les dernières maisons de la ville, disparaissant. ( $A$, p. 1106)

La seconde figure relève d'une composition iconique: la position d'un ou de plusieurs corps, souvent précisée dans les romans de Claude Simon. Au hasard citons les premières lignes des Géorgiques:

La scène est la suivante: [...] un personnage est assis devant un bureau, l'une de ses jambes à demi repliée sous son siège, le talon du pied soulevé, le pied droit en avant et à plat, le tibia formant avec la cuisse horizontale un angle d'environ quarante-cinq degrés, les deux bras appuyés sur le rebord du bureau (G., p. 649)

L'œil de l'auteur serait-il semblable à celui du géomètre-arpenteur RobbeGrillet? Un peu plus loin il justifie « la patiente et méticuleuse précision » par le dessin qui préside à l'exécution d'un projet (sculpture, architecture). On se souvient également des gravures galantes du genre de la "Fille Séduite » dans La Route des Flandres et la position du valet de chambre - " position d'un danseur au retombé d'un saut" ( $R F$, p. 250 et 251). La critique a toujours mis en relation la description très détaillée d'un objet ou d'une situation avec la nature d'un regard " appelé, aspiré, hypnotisé et comme envoûté par l'étude des choses ${ }^{7}$ ". Dans L'Acacia il y aurait au moins une " chose qui occupe la vision comme un gouffre où la vue $s^{\prime}$ engouffre ${ }^{8} n$ : celle du père mort,

6. On trouve dans L'Acacia, en particulier au milieu des scènes de combat et de peur, de courts moments lyriques (par exemple la perception du chant intermittent et non localisable du coucou, p. 1066-1068).

7. Voir p. ex. Dominique Viart qui renvoie aux études de Michel Deguy et de Roland Barthes, lesquelles autorisaient un rapprochement avec Robbe-Grillet (Une mémoire inquiète, PUF, 1997, p. 146-147).

8. M. Deguy, «Critique de la représentation », Critique, n 187, déc. 1962. 
les chaussures d'une taille toujours bizarrement démesurée, dessinant un V lorsque le corps est étendu sur le dos, ou encore parallèles, montrant leurs semelles cloutées [...] si le mort gît la face contre le sol, ou collées l'une à l'autre, ramenées près des fesses par les jambes repliées, le corps lui-même tout entier recroquevillé dans une position fotale (p. 1215)

Position hypothétique, reprise quelques pages plus loin dans une autre séquence comme si cette fois elle était authentifiée par le point de vue du narrateur:

Les genoux de l'un [des deux soldats blessés] sont écartés et ses talons joints, de sorte que ses jambes dessinent un losange, comme celles d'une grenouille. La jambe droite du second est repliée et son talon vient presque toucher le mollet de la jambe gauche allongée et raide. Sous cet angle on peut voir les semelles des souliers cloutés ${ }^{9}$. (p. 1218)

Il n'est pas certain que l'explication traditionnellement proposée par la critique soit ici pertinente. La précision géométrique de certaines descriptions renvoie plutôt à une mise à distance du pathétique, parfois par un soulignement quasi comique d'une situation intime.

Si la photographie fige l'image, par le texte ou le film, la composition formée par le ou les corps peut changer de forme, comme dans une figure de ballet, un exercice équestre ou un numéro de cirque. La figure dominante est celle de la position acrobatique du ou des corps, de l'équilibre instable et du déséquilibre. Cette figure équivoque, relevant de la beauté et en même temps de ce qui la menace jusqu'à la destruction, est fréquente dans les romans de Claude Simon: elle apparaît notamment dans des comparaisons et des métaphores appliquées aux situations périlleuses dans lesquelles se trouvent les cavaliers, ou dans les descriptions, méticuleusement distantes, des actes sexuels. Ainsi, pour ces dernières, dans La Bataille de Pharsale: " elle arquée son corps reposant seulement sur ses épaules et la plante abricot de ses pieds à plat sur le lit les reins soulevés les jambes dans la position de celles de ces acrobates faisant le pont " (BP, p. 641); ou bien: "O. cambre les reins de façon à amener son ventre au contact de l'autre ventre [...] ses jambes maintenant dans la position de celles d'une danseuse acrobatique exécutant la figure appelée le pont "(p. 702). Et dans Triptyque:

Entre chacune de ce que l'on pourrait appeler non pas des étreintes mais plutôt, en termes de boxe, des accrochages (lorsque l'un des adversaires à moitié assommé se cramponne à l'autre et lui paralyse les bras), et pendant lesquels les deux silhouettes sont confondues ${ }^{10}(T$, p. $805-806)$

9. Voir de même dans Le Jardin des Plantes la description de la photographie montrant deux déportés suppliciés $(J P$, p. 989-990).

10. L'épisode de la femme et de l'homme ivre interfere avec la prestation d'un clown. 
Si ces images contrastent avec la figure géométrique formée par les danseuses du Jardin des Plantes, elles ont pourtant en commun une prise de distance qui déshumanise les êtres:

Les attitudes hiératiques et stéréotypées rappellent celles de ces frises sculptées dont les personnages élèvent ou abaissent leurs bras, [...] les doigts joints, redressés et tendus en arrière, formant un angle droit avec les avant-bras, [...] cuisses et tibias dessinant un losange [...]. Les visages des danseuses sont totalement dépourvus d'expression, le regard vide, absent (JP, p. 992)

Mais la position acrobatique apparaît également dans une tout autre vision de l'amour, celle traditionnellement associée à la grâce aérienne des oiseaux ${ }^{11}$. On trouvera dans L'Acacia à plusieurs reprises le motif du ravissement, de l'extase amoureuse telle que se l'imagine la future épouse, mais dans un registre nettement moqueur, qui fait penser à Madame Bovary:

quelque chose l'attendait qui ressemblerait à une lévitation, quelque apothéose où elle se tiendrait, transfigurée et pâmée, portée sur un nuage soutenu par des angelots et d'où elle serait précipitée ensuite avec violence dans le néant. (p. 1082)

Dans un autre passage, la référence n'est plus mystique mais toujours acrobatique, le registre hors-temps restant le même:

comme si le photographe avait saisi ce fugace instant d'immobilité, d'équilibre, où parvenue à l'apogée de sa trajectoire et avant d'être de nouveau happée par les lois de la gravitation la trapéziste se trouve en quelque sorte dans un état d'apesanteur, libérée des contraintes de la matière, pouvant croire le temps d'un éblouissement qu'elle ne retombera jamais, qu'elle restera ainsi à jamais suspendue dans l'aveuglante lumière des projecteurs au-dessus du vide, du noir. (p. 1097-1098)

Derrière la référence aux jeux du cirque, il y a la mort, ce que montre la comparaison inattendue entre l'acrobate et le père contraint par un " art de mourir »:

non pas tant même de mourir que de le faire d'une certaine façon, c'est-à-dire (de même que l'acrobate ou la danseuse étoile revêtus de collants rapiécés transpirent et se désarticulent en coulisse au son d'un piano désaccordé [...] en vue du bref et fugitif instant d'équilibre instable, l'apothéose [...]) seulement de se tenir vingt ans plus tard debout [...] patientant jusqu’à ce qu'un morceau de métal lui fasse éclater la cervelle. (p. 1054)

Cette figure reliant explicitement la position acrobatique et la mort apparaît de manière très elliptique dans l'épisode du bordel, où le narrateur entend

11. Pour le rapprochement des oiseaux, de l'amour et de la lévitation acrobatique, voir B. Cendrars, Le Lotissement du ciel, le chapitre " Le nouveau patron de l'aviation ». Par exemple "nos deux colibris en tête à tête, chacun en extase devant la bouche de l'autre" (Gallimard, "Folio", p. 259). Les ornithologues précisent que ce sont les martinets noirs qui peuvent copuler en plein vol. 
sa propre voix, " enfin joyeuse, un peu rauque peut-être » - version comique du « coït aérien»:

« Mais non! Je ne te fais pas tomber!... Monte!... Bon Dieu, monte! Continue de monter!... Monte!... ", continuant à rire en même temps qu'à injurier pêle-mêle à voix basse l'espèce d'anachronisme équestre en train de brandir son sabre ${ }^{12}$ (p. 1241)

Le miracle d'équilibre est éphémère, il doit être suivi d'une chute. La scène du " coït aérien " permet de repérer, liée à la position acrobatique, une autre figure, la rupture d'équilibre, motif funèbre qui parcourt tout le livre: bustes des cavaliers oscillant d'avant en arrière, effort pour se tenir sur la selle d'un cheval quand les étriers ont été réglés pour un autre cavalier, course du soldat tombant sur le sol inégal sous le feu d'une mitrailleuse, étudiants en cubisme trébuchant dans les ornières d'une banlieue sinistre de Moscou, enfant essayant de faire tenir en équilibre sur une table les balles de fusil qu'il a déterrées, enfin mort du colonel, « le tout, cavalier, cheval et sabre basculant lentement sur le côté ${ }^{13}$ "(p. 1200).

Si le premier type de figuration des corps (apparition/disparition) appartient à un modèle littéraire inspiré par la musique (le lyrisme), le second, qui met en valeur l'équilibre instable, relève lui de la composition picturale ${ }^{14}$. Mais on pourrait tout aussi bien dire qu'il relève d'un modèle dramatique, dans les deux sens du terme. Différence essentielle: la beauté lyrique n'est pas suspecte, celle de l'équilibre instable est équivoque. La grâce n'est jamais loin de se défaire. Après le " coït aérien ", la jeune fille court et trébuche, et la description introduit des éléments disgracieux et déchirants - «se tordant les pieds ", "perdant l'un de ses souliers " - bien que la fin du paragraphe inscrive sa disparition dans un registre cosmique qui est aussi celui du lyrisme. S’il est vrai que dans L'Acacia la scène du "coït aérien " est unique, on voit bien que par certains traits relatifs à la figuration des corps elle évoque d'autres scènes. Comme celle où l'auteur imagine comment une femme vient sur la plage, à la fin de l'été 1914, annoncer la mort du capitaine (p. 1139-1140): elle court, se tord les talons, abandonne ses chaussures. La description exaspérée - la présentation dans une longue phrase, les

12. Sur le télescopage des scènes sexuelles et des scènes de guerre, voir N. Piégay-Gros, «Une vulgaire histoire de cul entre une putain et deux imbéciles ", Cabiers Claude Simon, n 8, 2013, p. 53-63.

13. Emblème récurrent dans La Route des Flandres, La Bataille de Pharsale, Le Jardin des Plantes.

14. On pense au tableau de Paul Klee Équilibre instable, mais aussi à ce que Mallarmé, dans un hommage à Théodore de Banville, appelait "l'éventement de la gravité " (Divagations, dans Euvres complètes, Gallimard, « La Pléiade », t. II, p. 143). 
précisions données par étapes, l'intensité des gestes - le mélange d'élégance et de vulgarité, permettent un rapprochement avec notre scène de séparation. L'apparition progressive au-dessus de la ligne des dunes répond à la disparition progressive de la fille en robe rouge, et de même on note que les éléments disgracieux, voire grotesques - sa gesticulation est celle d'une " folle ", d'un " pantin désarticulé " ou d'une "boiteuse »- surviennent au moment le plus tragique. Mais cette fois encore la scène est traversée par des notations de bruits et d'éléments du paysage qui lui donnent une beauté qui aggrave et qu'aggrave la déchirure. Et le fracas des vagues déferlantes et les cris des oiseaux qui couvrent la voix enrouée, indifférents à l'annonce funèbre, font écho à une autre scène, celle qui se passe dans la ville d'eau, quand l'épouse reçoit l'annonce de la mort de son mari (p. 1183-1184). De nombreux aspects sont identiques: la certitude instantanée que tout à jamais prend fin, le ralenti et la suspension du temps, le contraste entre l'horreur que suscite "l'automobile, l'espèce de monstre, de coléoptère ${ }^{15}$ aux ailes de tôle, aux yeux exorbités, aux cuivres jaunes " et l'agrandissement cosmique, la beauté de la nature environnante indifférente au malheur qu'elle redouble. Seule différence: dans la ville d'eau la douleur est muette (les lèvres ne peuvent articuler aucun son: "comme un poisson hors de l'eau, à demi asphyxié, continue à ouvrir et fermer la bouche sur du vide ", "le même hurlement muet, déchirant "), l'autre est au contraire très sonore (les cris, le bruit des vagues).

\section{DE LA CHOSE VUE À L'ALLÉGORIE}

Posture des corps où l'un tient à bout de bras le corps arqué de l'autre, la garde miraculeusement en équilibre tandis que les bouches se joignent, jusqu'à ce que le train prenne de la vitesse, que la pesanteur la rejette sur le sol et qu' en courant elle trébuche sur le ballast. Jouant sur le double sens du verbe " regarder ", on se demandera en quoi cette scène regarde notre auteur.

Il est tentant de confronter les adieux d'août 1939 et ceux d'août 1914. Proposée à deux reprises, la relation de ceux du père qui s'appuie sur la mémoire des deux cousines (enfants elles ont assisté à son départ) est surtout fictive et donne une représentation qui a peu à voir avec celle de 1939. La séparation a lieu dans des conditions différentes: le père tout harnaché quitte

15. Métaphore à rapprocher de la vision du train, "les flancs brunâtres des wagons ", " peints d'une couleur marron écaillée ", « le bois marbré d'écailles " (p. 1101-1102). 
à cheval la cour de la maison, en présence d'un groupe (la famille, la bonne noire, la concierge, l'ordonnance du capitaine). Mais c'est la figuration des corps qui attire l'attention. Claude Simon a d'abord imaginé auparavant un ultime rapport sexuel:

la serrant contre lui, et elle raidie, sans voix, glacée - ou peut-être l'attirant une dernière fois sur elle avec une sorte d'avidité sauvage, s’ouvrant à lui, le recevant, cramponnée, déjà folle, hoquetante, dans une furieuse protestation, une furieuse, déchirante et ultime étreinte, et maintenant rigide de nouveau, l'œil sec (p. 1141)

Si l'épithète "sauvage » est commune aux deux scènes d'adieux, la présence des termes "cramponnée ${ }^{16}$ ", " hoquetante ", "furieuse étreinte ", qui figuraient dans les lignes relatives à la foule mais exclus de la scène du « coït aérien ", indique assez la différence. Et surtout la suite du texte insiste plusieurs fois sur l'absence de larmes et l'attitude rigide de l'épouse au moment du départ. Deux métaphores sont remarquables, la sculpture et le théâtre. La relation qui est donnée au chapitre VII (p. 1141-1145) a retenu essentiellement la première en évoquant l'allure de la femme noire qui tient l'enfant, " quelque statue venue du fond des âges ${ }^{17}$ ", " une colonne de marbre », et la position de l'épouse au milieu d'un "groupe de personnages " "serrant dans ses mains l'appui du balcon». Métaphore plus explicite dans le chapitre IX (p. 1176) qui la montre «cramponnée à l'appui » du balcon de la véranda " au premier rang [...] comme dans une loge de théâtre ». Cette mise en scène implique en même temps un modèle esthétique (plan fixe de la composition de groupe) et une distance de l'auteur qui ne tient pas seulement à son trouble - évoquer l'intimité de ses parents - mais aussi à l'émotion d'imaginer une situation où il était présent (dans les bras de la bonne noire) mais dont il ne possède évidemment aucun souvenir: il avait moins d'un an.

Claude Simon a-t-il réellement été témoin de la scène d'août 1939, s'estil inspiré de documents? Si pour 1914 les dessins et les photos de la gare de l'Est abondent, ce n'est pas le cas pour $1939^{18}$. Le regard entièrement

16. Si le mot est présent dans la description du couple, c'est dans une construction négative.

17. La "passive ténacité de statue" que Claude Simon prête à la mère (p. 1176) est évidemment confortée par la pose photographique et la mode vestimentaire (robes entravées, corsages baleinés). Sur la famille au balcon, voir la note de l'édition "La Pléiade " (E II, p. 1594).

18. Quelques rares photos sont visibles sur la toile ou dans des ouvrages historiques. Également peu de témoignages littéraires sur ces journées de mobilisation en août-septembre 1939 (Aragon, Simone de Beauvoir), contrairement à août 1914 (largement évoqué dans plusieurs romans, dans Les Thibault, L'été 14, de Roger Martin du Gard, Le Sang noir de Louis Guilloux, et le grand tableau du peintre 
focalisé sur la femme et son éloignement progressif implique le point de vue de celui qui est dans le train. Aurait-il été l'acteur? Dans la séquence du 27 août, n'est pas mentionnée la femme qui l'accompagnait à la gare (à laquelle Claude Simon fait allusion ailleurs, qu'il épousera plus tard à l'occasion d'une permission), celle qui a enveloppé les sandwiches dans du papier de soie - ce qui provoque son irritation -, modèle dans une académie de peinture et qui, plus tard, suivra des cours de dactylo pour taper ses manuscrits. L'émergence du mot « dactylo » - la jeune fille en robe à fleurs rouges étant " peut-être " une " dactylo » - qui avait jadis une connotation érotique (depuis Apollinaire dans "Zone») autoriserait-t-elle à superposer les images des deux jeunes femmes? Non, si l'on se réfère à ce que Simon a écrit dans La Corde raide: la jeune femme qui l'accompagnait «a réussi à se tenir convenablement jusqu'au dernier moment ${ }^{19}$ ".

Une scène identique apparaît dans Histoire:

ses yeux agrandis immenses me fixant mais pas de pleurs [...] et elle et moi toujours debout l'un devant l'autre la machine lâchant et retenant sa vapeur [...] et rien que nous deux debout dans ce silence, comme si le monde entier était mort, englouti, comme si plus rien n'existait, sauf son visage, et même pas le flanc verdâtre du wagon derrière, même pas le col de son manteau, ses cheveux, ou le front, la bouche: seulement les yeux - et tout à coup, sans transition, cessant de la voir, quoique je n'aie pas tourné la tête, tandis que tout le brouhaha refluait de nouveau, le halètement de la locomotive, les gens, les roues des chariots, et de nouveau rien encore que ce silence, comme si on avait posé un bâillon sur tout, coupé... (p. 168)

et plus loin :

Je dis Tu sais bien que nous ne pouvons pas nous perdre tu sais bien Là-bas au bout du quai la machine haletait régulièrement Yeux immenses me regardant humides mais pas de Il s'ébranla dans un bruit d'attelles tiraillées entrechoquées commença à prendre de la vitesse je criai Hélène! elle rentra la tête Tout de suite à la sortie de la gare les voies commençaient à tourner les flancs lisses métalliques des wagons nus se succédaient pivotaient comme un mur vert foncé (p. 361)

La comparaison des versions de La Corde raide, d'Histoire et de L'Acacia montre des différences saisissantes. Elle donne à penser que si l'origine de la

américain Albert Herter Le Départ des poilus, août 1914). Le thème de recherche par Internet " adieux en gare " permet de découvrir des images plus récentes dont deux ou trois montrent une femme comme rivée au train dans une position acrobatique.

19. $C R$, p. 140. Le narrateur fait allusion à la nuit qu'il a passée avec elle la veille de son départ et relate le voyage en train. 
scène est autobiographique ${ }^{20}$, la version de L'Acacia construit une fiction quasi allégorique qui illustre la séparation amoureuse exacerbée sur fond de mort.

Il faut en venir à la robe rouge, absente des autres versions, que nous voyons et qui nous regarde. On n'exclura pas la mémoire d'une affiche cinématographique - «l'affiche violente, ses personnages aux postures violentes, passionnées, ses couleurs violentes ${ }^{21}$ »- mettant l'accent sur le côté érotique-spectaculaire de la scène: comme la posture de Rita Hayworth sur l'affiche de L'Amour vient en dansant, ou les robes rouges qu' elle porte dans L'Affaire de Trinidad, Les Amours de Carmen ou La Belle du Pacifique ${ }^{22}$. L'expression "au visage peinturluré de putain » attire l'attention, par son côté équivoque, brutal mais pas entièrement inattendu: la posture "cambrée » et la robe "d'un tissu voyant, bon marché " pouvaient l'induire. La mention de la robe rouge renvoie évidemment à la Corinne de La Route des Flandres, "sa vaporeuse et indécente robe rouge oscillant, se balançant au-dessus de ses jambes » (p. 208). Ce texte apportait plus loin une précision, à savoir que le rouge par un temps ensoleillé est peut-être inventé:

debout dans le contre-jour ensoleillé d'une fin d'après-midi ${ }^{23}$, dans cette robe rouge couleur de bonbon anglais (mais peut-être cela aussi l'avait-il inventé, c'est-à-dire la couleur, ce rouge acide, peut-être simplement parce qu'elle était quelque chose à quoi pensait non son esprit, mais ses lèvres, sa bouche, peut-être à cause de son nom, parce que "Corinne " faisait penser à " corail »?...) $(R F$, p. 355)

Dans ce contexte, la robe rouge est vulgairement associée au désir masculin. Mais la couleur ouvre toujours divers horizons. Tant il est vrai, comme l'écrivait Merleau-Ponty, que si " la robe rouge tient de toutes ses fibres au tissu du visible ", elle touche en même temps "à un tissu d'être invisible 24 ", et l'invisible de Simon - comme celui de Proust - peut toucher à des constellations diverses. Un lecteur de Proust ne pensera pas à la couleur rouge de la robe en vieux satin que portait la duchesse de Guermantes et quadmire

20. Mais avec des variantes. Dans Histoire c'est la femme qui part (pour Barcelone?). Les yeux « humides mais pas de " [larmes] confirmeraient la version de La Corde raide.

21. Le Vent, p. 68.

22. Films distribués en France après la guerre et au début des années 1950. Seuls les deux derniers sont en technicolor. Mais les affiches sont en couleur.

23. C'est également en fin d'après-midi que se promène la jeune femme en rose dans les ruines de Berlin "d'un rouge noirci, couleur de briques cuites ", tandis que descend peu à peu "le disque du soleil d'un rouge orangé " (Le Jardin des Plantes, p. 1019-1020). Précisons que de nombreuses femmes en rouge, rose, géranium, cerise, corail, etc., traversent les romans de Claude Simon.

24. M. Merleau-Ponty, Le Visible et l'invisible, Gallimard, "Tel », 1979 [1964], p. 174. Merleau-Ponty ajoute plus loin: «Comme l'ont dit beaucoup de peintres, je me sens regardé par les choses » (p. 183). 
tant le narrateur de La Prisonnière ("vous aviez l'air d'une espèce de grande fleur de sang, d'un rubis en flammes ${ }^{25}$ ); mais au visage de la tenancière du petit kiosque des Champs-Élysées, là où la grand-mère a son attaque cérébrale: le maquillage outrancier, mal fait, presque clownesque, appelle l'idée de la mort ${ }^{26}$. Dans ces pages de L'Acacia, la couleur prend place dans une série de figurations de visages, comme maquillés: le chauffeur et le mécanicien " aux joues noircies de suie, aux yeux bordés de rouge »; les visages des vieilles femmes " crevassés de rides, jaunes "; " les masques desséchés des vieilles vêtues de noir ". Celui qui dans L'Acacia voit la robe rouge est aussi celui que désigne le "il » de la formule répétée en début d'alinéa un peu plus loin: "Et maintenant il allait mourir ». Le terme " putain » est le signe de l'intensité du désir du narrateur: comme la robe rouge, c'est un adieu au plaisir charnel promis par un être à la beauté simple et éclatante, ce sur quoi insistaient les lignes précédentes. Et on aura compris que dans l'échelle des privations celle-là, jointe à l'idée de la mort irrémédiable, est sans doute la plus cruelle 27 .

Lidée douloureuse de la privation invite à un rapprochement avec un passage évoquant le parcours du régiment du père vers la gare ${ }^{28}$ :

suivant les étroites ruelles de la ville haute, passant devant les vieux hôtels de briques, la halle médiévale, les cafés aux terrasses fleuries d'hortensias en caisses et décorés de femmesiris, le balcon du cercle où les vieux messieurs arrachés pour un moment à leurs tables de bridge et à leurs rocking-chairs applaudissaient de leurs mains parcheminées, leurs faibles voix couvertes par les vivats aigus des cocottes décolletées penchées à leurs côtés, offrant comme dans des corbeilles leurs seins éblouissants, leurs lèvres ouvertes sur les humides grottes roses de leurs bouches aux dents éblouissantes, et jetant des fleurs. (p. 1040)

La direction du texte, contrairement au trajet, est imprévisible: l'énumération descriptive sort du cadre en faisant proliférer un détail et dériver le propos. À partir de la mention du balcon où applaudissent les vieux messieurs,

25. M. Proust, La Prisonnière, À la recherche du temps perdu, Gallimard, "La Pléiade ", 1987-1989, t. III, p. 547.

26. « son museau énorme et irrégulier enduit de plâtre grossier, et son petit bonnet de fleurs rouges et de dentelle noire surmontant sa perruque rousse » (Le Côté de Guermantes I, éd. cit., t. II, p. 605). Fautil rappeler que c'est à proximité du kiosque qu'a eu lieu entre le narrateur et Gilberte un jeu sexuel d'une grande intensité? (À l'ombre des jeunes filles en fleurs, éd. cit., t. I, p. 485).

27. On retrouve le même sentiment de perte cruelle dans le célèbre poème de René Char "Le loriot " (dont l'exergue précise « 3 septembre 1939 ») : Le loriot entra dans la capitale de l'aube./ Leépée de son chant ferma le lit triste./ Tout à jamais prit fin. "

28. Deux passages de L'Acacia décrivent ce parcours. Celui de la page 1077 se contente d'énumérer les lieux traversés et les éléments du décor urbain. 
la série prend un cours inattendu: pour en arriver au geste patriotique et délicat (jet de fleurs) elle passe par des détails focalisés sur l'objet sexuel. Si les vieux messieurs ne seront pas privés des plaisirs que leur offrent leurs " cocottes ", ce n'est évidemment pas le cas de ceux, plus jeunes, qui partent à la guerre. Cette vision hante l'imagination de l'auteur: dans Le Tramway (p. 1284-1285) il reviendra sur la fascination exercée par les " poules » des graves messieurs du Cercle (mêlées aux héroïnes de cinéma aux poitrines débordantes comme sur les couvertures de magazines).

\section{POUR CONCLURE}

Tout donne à penser que dans cet épisode dont l'origine est probablement autobiographique, la scène du "coït aérien " est au moins en partie inventée. Elle dépasse suffisamment le statut de chose vue pour qu'on puisse lui prêter un sens allégorique: elle veut donner une idée violente de la beauté mise à l'épreuve d'une perte cruelle, le corps de la femme aimée, et d'un destin dont l'auteur pense qu'il va répéter celui du père. En même temps la scène révèle la complexité d'une écriture marquée par la distance et la luxuriance. Distance qui elle-même change dans le roman selon le motif et l'angle de vue, nulle dans certains segments lyriques, ailleurs rendue sensible par une précision impitoyable qui ôte toute vibration ou par une référence explicite aux compositions iconiques. Luxuriance qui tient autant à la diversité de ses sources (photographies, récits familiaux, entrevisions personnelles) qu'à son aptitude à jouer à la fois de la fragmentation qui fait se succéder les unités d'un ensemble et de la ressemblance obtenue par la récurrence des emblèmes et des motifs. La scène d'adieux, popularisée par le roman et le film, appelle la beauté. Qu'on pense aux phrases de Flaubert relatant la dernière entrevue de Frédéric Moreau avec Madame Arnoux ou à la manière dont Jacques Demy a filmé la séparation des amants dans la gare de Cherbourg - sans oublier la musique de Michel Legrand qui amplifie l'émotion ${ }^{29}$ - la beauté est au rendez-vous. Comme dans les entrevisions de Proust (la jeune fille de la gare sur le trajet de Balbec) ou de Simon (la fille en rose dans les ruines de Berlin ${ }^{30}$ ), ou dans la Gradiva de Jensen commentée par Freud, la démarche de la jeune femme dans les ruines de Pompéi, dans la lumière de midi, heure chaude et sacrée. La mort est quelque part, derrière,

29. La séquence est en ligne sur youtube.

30. Respectivement, À l'ombre des jeunes filles en fleurs, Le Jardin des Plantes. 
à côté de la beauté. C'est aussi ce qui apparaît dans la séquence de la fille en rouge. En son temps Christian Prigent regrettait que L'Acacia soit de « la belle ouvrage ${ }^{31}$ ». Il faut le reconnaître: Claude Simon n'a jamais pratiqué le culte de la laideur ni l'art de l'informe, tellement à la mode. Il est de ceux qui ne considèrent pas la beauté comme une distraction ou pire une imposture. L'auteur de L'Acacia, par son art du montage et ses figures allégoriques et poétiques s'inscrirait-il dans la tradition des écrivains qui, pour reprendre les termes de D.H. Lawrence dans "Chaos in poetry ", se protègent du chaos par une recherche d'art qui les oppose à ceux qui restent au contact de la déchirure ${ }^{32}$ ? L'Acacia présente pourtant l'une des diverses manières que possède un écrivain de " rester joint à son trouble " pour reprendre les mots de Michaux ${ }^{33}$. Libre au lecteur grincheux d'en regretter l'excès de beauté.

31. Nous renvoyons au commentaire de L'Acacia que le poète Christian Prigent a fait paraitre en 1990 dans la revue $T X T, \mathrm{n}^{\circ} 25$, sous le titre «La belle ouvrage ». Contrairement à C. Prigent, je crois que l'humour est bien présent, plusieurs de nos citations l'attestent.

32. Lawrence reconnaît que l'artiste est guidé en même temps par l'appétit du chaos et la peur du chaos. Cet article a été traduit dans le numéro spécial des Cahiers de l'Herne qui lui a été consacré (1988, p. 189-191).

33. C. Prigent cite la formule de Michaux ("Observations ", Passages, dans Euvres complètes, Gallimard, "La Pléiade ", 2001, t. II, p. 348). 